\title{
MONITORIZAÇÃO DA QUALIDADE EM SAÚDE: ESTUDO DA SATISFAÇÃO DOS UTENTES
}

\author{
Rosa Martins ${ }^{1}$ \\ Cecília Frutuoso ${ }^{2}$
}

\begin{abstract}
Resumo:
Enquadramento: A satisfação dos utentes com as instituições de saúde apresenta-se na atualidade como um indicador fundamental na determinação dos aspetos a melhorar e a aperfeiçoar de forma prioritária ou seja na implementação de parâmetros contributivos para a qualidade dos serviços prestados. Por essas razões torna-se imperioso o conhecimento dos níveis e determinantes da satisfação dos utilizadores com vista à excelência dos cuidados.

Objetivos: Avaliar a satisfação dos utentes de um Centro de Saúde da região Centro de Portugal e verificar associações existentes entre esta satisfação e algumas variáveis sociodemográficas, clínicas e psicossociais.

Métodos: Trata-se de um estudo não experimental, transversal, descritivo correlacional, de caráter quantitativo, que envolveu 303 utentes do Centro de Saúde. Para o efeito foi utilizado um questionário que contém variáveis sociodemográficas, clínicas e psicossociais e ainda a escala "EUROPEP” para avaliar o grau de satisfação

Resultados: Constatámos que a satisfação global da amostra é elevada uma vez que oscila entre um valor mínimo de 63 e um máximo de 275, correspondendo-lhe uma média de 218,92. Verificámos ainda que a dimensão com maiores níveis de satisfação (85.6\%) dizem respeito à relação/comunicação, e inversamente os utentes estão mais insatisfeitos com a organização do serviço. Os utentes com maiores rendimentos mensais e que percecionam maiores benefícios nos programas de reabilitação são também aqueles que se apresentam mais satisfeitos.

Conclusão: $O$ nosso estudo reforça o paradigma de que a satisfação dos utentes com os cuidados prestados tem um caráter abrangente e multidimensional, depende de fatores também variáveis no tempo, no
\end{abstract}

1 Professora Coordenadora da Escola Superior de Saúde de Viseu. Email: rmartins.viseu@gmail.com

${ }^{2}$ Enfermeira Especialista em Enfermagem de Reabilitação no Centro de Saúde da Guarda. 
espaço e nas populações, tratando-se portanto de um processo dinâmico e subjetivo em constante construção.

Palavras-chave: Satisfação, Utentes, Centro de Saúde, Reabilitação, Qualidade.

\begin{abstract}
:
Background: The satisfaction of users with health institutions is nowadays as a fundamental indicator in determining the aspects to be improved and improved as a priority or in the implementation of parameters contributing to the quality of services provided. For these reasons it is imperative to know the levels and determinants of user satisfaction for excellence of care.

Objectives: To evaluate the satisfaction of the users of a Health Center in the central region of Portugal and to verify associations between this satisfaction and some sociodemographic, clinical, and psychosocial variables.

Methods: This was a non-experimental, cross-sectional, correlational descriptive study of a quantitative nature involving 303 patients from the Health Center. A questionnaire containing sociodemographic, clinical and psychosocial variables was used and a "EUROPEP "To assess the degree of satisfaction

Results: We found that the overall satisfaction of the sample is high since it oscillates between a minimum value of 63 and a maximum of 275, corresponding to an average of 218,92 . We also verified that the dimension with higher levels of satisfaction (85.6\%) Relate to the relationship / communication, and conversely the users are more dissatisfied with the organization of the service. The users with the highest monthly income and who perceive greater benefits in rehabilitation programs are also the ones that are more satisfied.Conclusion: Our study reinforces the paradigm that the satisfaction of the users with the care provided has a comprehensive and multidimensional character, depends on factors also variable in time, in space and in the populations, being therefore a dynamic and subjective process in constant construction.
\end{abstract}

Keywords: Satisfaction, Users, Health Center, Rehabilitation, Quality.

\title{
INTRODUÇÃO
}

De acordo com o Dicionário da Língua Portuguesa Contemporânea (2001), o termo "Satisfação" tem a sua origem no Latim satisfactione definido como "o ato ou efeito de satisfazer ou satisfazer-se". Na opinião de Cerqueira (2012, p.9), "a satisfação está diretamente associada ao facto do indivíduo obter, ou não, aquilo que anseia” (...) e decorre da resposta positiva que o indivíduo obtém em relação às suas necessidades. Ribeiro (2005,citado por Duarte,2012, p.22) diz-nos que “...é o primeiro sujeito que atribui o sentido da satisfação em função da realização das 
necessidades percebidas, das expectativas e dos resultados obtidos. Este processo pressupõe elementos afetivos e cognitivos individuais, uma vez que se trata de aspetos determinados por cada pessoa". Contudo, na perspetiva veiculada por Ferreira (2014), a satisfação pode ser entendida como uma atitude, uma emoção ou um sentimento que é passível de ser medido e verbalizado através de uma opinião. Nesta condição, a satisfação incorpora aspetos afetivos, cognitivos, avaliativos e até comportamentais.

A satisfação que diz respeito aos utentes com os cuidados de saúde, apesar de já ter ser sido investigada por diversos autores, está ainda longe de se apresentar como tema consensual. Na verdade, Godinho (2011) apresenta a satisfação como sendo um conceito subjetivo uma vez que assume diversos significados consoante o contexto em causa. Acrescenta que qualquer entidade que preste cuidados de saúde visará a satisfação das necessidades objetivas e/ou subjetivas como alvo, tanto dos utentes utilizadores efetivos como dos utentes potenciais. Estes pressupostos levam-nos a subscrever o que diz Costa (2011), ao afirmar que se trata de um conceito dinâmico, subjetivo e com forte valor cultural sofrendo mutações constantes. Johnson (2001, citado por Vilares e Coelho 2005, p.78) diz-nos que "a satisfação pode ser definida com base em dois pressupostos distintos: 1) a satisfação como sendo um juízo avaliativo que se faz após a escolha relativamente a uma compra ou a uma transacção específica; 2) a satisfação como sendo um processo cumulativo, representando uma avaliação global que é baseada na experiencia adquirida ao longo do tempo".

Ferreira (2014), refere que a opinião do utente tem vindo a ser cada vez mais considerada no domínio científico e pelos vários parceiros sociais no sentido monitorizar a qualidade dos serviços de saúde e de avaliar a eficácia das necessidades corretivas que se têm vindo a implementar no Sistema Nacional de Saúde. Contudo, o conceito de satisfação dos utentes apesar de amplamente discutido, não tem sido devidamente definido e clarificado, quer pelos investigadores, quer pelos utentes quando questionados. Apesar das dificuldades descritas, a satisfação do cliente assume uma importância fundamental como medida dos cuidados de saúde porque nos dá uma informação acerca do sucesso dos prestadores, em relação aos valores e às expectativas dos seus clientes (Cerqueira, 2012). Nesta perspetiva e de acordo com Marques (2010, p.52), "a satisfação dos utentes é assim considerada um indicador importante na monitorização da qualidade dos cuidados de saúde prestados”. Esta visão tem sido aceite por variados autores incluindo 
Duarte (2012), ao dizer-nos que hoje reconhece-se que as perspetivas dos doentes são largamente vistas como determinantes na avaliação da qualidade dos cuidados prestados. $\mathrm{O}$ aumento do consumo de serviços de saúde, e consequente aumento da responsabilidade das organizações de saúde, levou à necessidade das organizações de saúde saberem se os seus utentes ficavam ou não satisfeitos com os serviços por eles prestados. Além disso como aponta Ferreira (2014, p.7), "as opiniões vão ser alicerce para a implementação de mudanças nas organizações, permitindo assim responder às expectativas e necessidades das populações no campo da saúde”. Costa (2011) enfatiza o facto de as instituições valorizarem as avaliações dos cuidados feitos pelos utentes, no sentido de só assim se poder garantir a qualidade nos cuidados de saúde. Acrescenta que para a monitorização da qualidade dos serviços de saúde, no sentido de identificar problemas passíveis de corrigir, criar novas expectativas relativamente aos cuidados e por fim, a reorganização dos serviços propriamente dito, é indispensável ter como base a opinião dos utentes. Para além disso, Arakawa et al. (2012) acrescentam o facto de que a satisfação dos utentes com atendimento pode ainda contribuir para a melhoria da sua qualidade de vida pois parece promover a adesão ao tratamento e a continuidade na utilização dos serviços.

Perspetivando a satisfação dos utentes no sentido dos cuidados de Enfermagem e de acordo com as diretrizes emanadas pela Ordem dos Enfermeiros (OE) (2011), na procura permanente de excelência no exercício profissional, o enfermeiro persegue os mais elevados níveis de satisfação. Acrescenta que o profissional de enfermagem nas suas intervenções deve elevar a forma constante da abordagem e o respeito pelo utente a vários níveis (capacidades, crenças....), não descurando o envolvimento no planeamento de todo o processo de doença, sempre com o objetivo de minimizar os aspetos mais negativos. Apesar de no passado, o paciente ter sido desvalorizado como ponto central na prestação dos cuidados de saúde, ao longo dos tempos esta questão tem vindo a mudar,tornando-se o figurante central.De facto o utente assume hoje o papel principal colaborando na avaliação da satisfação, constituindo-se esta uma importante ferramenta de pesquisa e de planeamento nos cuidados de saúde e de enfermagem.

Sabemos que há uma diversidade bastante grande de fatores que podem influenciar o grau de satisfação das pessoas: de entre estes destacam-se os atributos do próprio individuo, as experiencias vividas anteriormente, as variáveis do contexto onde os indivíduos estão inseridos e as próprias expectativas sobre as diferentes dimensões da satisfação. Compreendemos que devido à sua natureza multidimensional, se torna 
difícil obter uma definição consensual de satisfação bem como a sua própria operacionalização.

Para Costa (2011), existem 12 dimensões de satisfação relacionadas com os cuidados de saúde que correspondem: 1) elementos respeitantes a admissão; 2) acolhimento; 3) prestações hoteleiras; 4) conforto; 5) higiene das instalações; 6) visitas; 7) ocupação dos tempos livres; 8) apoio para os cuidados básicos; 9) relações interpessoais; 10) alta; 11) competência; 12) estado de saúde do utente.

Oliveira (2012), numa visão mais generalizada, defende a existência de 4 aspetos principais que na sua opinião são determinantes na satisfação: (1) Característica dos pacientes - inclui as variáveis sociodemográficas, as expectativas destes sobre a consulta médica e os seus estados de saúde; (2) Características dos profissionais que prestam o atendimento (incluindo traços de personalidade, qualidade técnica e a "arte do cuidado"); (3) Aspetos da relação médico/enfermeiro - paciente; (4) Fatores estruturais e ambientais (incluindo o acesso, forma de pagamento, tempo de tratamento, marcação de consulta e outros). Posteriormente, o mesmo autor justifica a inclusão de novos fatores afirmando que a crescente exigência dos utentes dos serviços de saúde fez com que determinados fatores, como a informação, a comunicação, a qualidade das instalações e o desempenho dos profissionais de saúde fossem destacados e também relacionados diretamente com o processo de satisfação. Alguns dos fatores apontados incluem características e atributos pessoais relativos aos utentes, bem como, as experiencias anteriores, as variáveis do contexto e as expectativas dos utentes sobre as diferentes dimensões da satisfação. Dentro destas variáveis de contexto, Lopes (2013) diz-nos que a perceção das pessoas sobre a funcionalidade da sua família também se tem revelado como fator preditivo da satisfação geral e por isso deverá ser incluída nos protocolos de avaliação.

Em síntese, sabemos que uma das preocupações crescente e atual nos serviços de saúde, é a qualidade dos cuidados em saúde, e corroboramos a ideia de que a avaliação da satisfação dos utilizadores se torna um pilar absolutamente fundamental das políticas de saúde.

\section{MATERIAL E MÉTODOS}

Tendo em conta a magnitude do problema, a sua transcendência e o nosso interesse em aprofundar esta problemática, optámos por um estudo quantitativo, não experimental, transversal e descritivo-correlacional alicerçado na seguinte questão de investigação: Qual o grau de satisfação 
dos utentes que recorrem ao Centro de Saúde e em que medida as variáveis sociodemográficas, clinicas e psicossociais interferem nessa satisfação? Com o intuito de responder à questão de investigação, definimos como objetivos do estudo: Conhecer o grau de satisfação dos utentes que recorrem ao Centro de Saúde; Identificar a funcionalidade percecionada pelos utentes; Averiguar a existência de relação entre variáveis sociodemográficas, clinicas e psicossociais e a satisfação dos utentes.

A amostra utilizada é do tipo não probabilístico por conveniência, constituída por 303 utentes do Centro de Saúde. Os critérios de elegibilidade adotados foram: Utentes que recorrem ao Centro de Saúde com médico de família atribuído. Já os critérios de exclusão incidiram sobre os utilizadores dos cuidados de saúde de forma espontânea/ocasional (que auferem dos cuidados por estarem de férias, de passagem, etc ...).

Tendo em conta a natureza do problema de investigação, os objetivos do estudo e as características da amostra, decidimos efetuar a recolha dos dados recorrendo a um questionário que permite avaliar a satisfação dos utentes do Centro de Saúde. Neste sentido, utilizamos como instrumento de medida destes cuidados um questionário composto por questões por nós formuladas e uma escala - o EUROPEP - validada para a população portuguesa por Ferreira, Raposo e Godinho (2005), que foi construída para fornecer feedback para a melhoria da prática, desempenho e organização dos cuidados dos profissionais de saúde. Assim, a primeira parte do instrumento de colheita de dados procura informações sobre os utilizadores (dados sociodemográficos, clínicos e contextuais, e familiares, tendo em conta a sua funcionalidade). A segunda parte inclui o questionário EUROPEP que é composto por (1) Indicadores chave que inclui as dimensões: relação e comunicação, cuidados médicos, informação e apoio, continuidade e cooperação, e, organização dos serviços, (2) Indicadores de áreas específicas de satisfação (consulta, marcação e acessibilidade, características dos profissionais, condições do centro de saúde e os serviços prestados). Para além destes indicadores foram incluídos no questionário perguntas abertas no sentido dos utentes se expressarem sobre as melhorias que entendem ser fundamentais para o bom funcionamento do Centro de Saúde bem como aspectos agradáveis e desagradáveis que encontram nas suas visitas à instituição. A colheita de dados foi efetuada nos meses de janeiro, fevereiro e março de 2015.

Todos os procedimentos foram efetuados segundo uma rigorosa conduta ética, garantindo-se o anonimato e confidencialidade dos dados recolhidos. O tratamento estatístico da presente investigação foi efetuado 
através do programa Statistical Package for the Social Science (SPSS), versão 20.0 para o Windows e Word Microsoft.

\section{RESULTADOS}

A amostra do estudo é constituída por 303 participantes, 219 do sexo feminino e 84 do sexo masculino. A idade da amostra total oscila entre 16 (min) e 84 (máx.) anos com uma média de 46,23 anos, Dp=16,25 e CV= $35,15 \%$, o que nos indica a existência de uma elevada dispersão em torno da média. A média de idades para o sexo feminino $(M=42,36)$ é inferior à do sexo masculino $(\mathrm{M}=56,31)$.

A maioria dos participantes $(75,9 \%)$ vive acompanhado, reside em meio urbano (74,6\%) e tem 6 anos de escolaridade (35,3\%). Em termos profissionais $42,9 \%$ estão empregados, $27,1 \%$ reformados e $22,1 \%$ desempregados. A maioria (60,1\%) aufere valores mensais acima dos 500 euros, seguindo-se o grupo $(23,9 \%)$ que dispõe de valores que se situam entre 250 e 500 euros.

Nas deslocações ao Centro de Saúde, a maioria $(79,5 \%)$ utiliza carro próprio, gasta em média cerca de $15 \mathrm{mn}$ no percurso e o tempo de espera para serem atendidos no Centro de Saúde oscila entre $0-66 \mathrm{mn}$ com uma média de $10 \mathrm{mn}$ e $\mathrm{Dp}=16,23$.

Em termos clínicos, 85,4\% são portadores de doenças prolongadas (mais de três meses) e as patologias mais presentes são a HTA $(29,0 \%)$, diabetes (14,3\%), problemas digestivos (12,9\%) e patologias osteoarticulares $(11,6 \%)$. Os programas de reabilitação são frequentados por $41,4 \%$ dos utentes da amostra (sobretudo mulheres - 23,6\%), sendo fundamentalmente realizadas no hospital $(62,9 \%)$ e os benefícios que lhe reconhecem são apenas moderados (34,3\%). Observámos que 39,4\% veio à consulta por iniciativa própria seguindo-se a consulta previamente combinada com o médico (29,8\%) e os motivos que estiveram na base da consulta são: consultas de rotina programadas (52,8\%), alívio da dor $(6,9 \%)$ e outras (consulta de planeamento familiar, tratamentos, vacinação, baixa médica, receituário e cheque dentista).

Sobre a forma de marcação da consulta, 31,7\% diz que foi marcada no próprio Centro de Saúde, 19,8\% marcação na consulta anterior, e apenas $17,5 \%$ refere o telefone. As marcações efetuadas por terceiros $(3,0 \%)$ ou por convocatória (2,6\%) têm pouca expressão nesta amostra. Perante a possibilidade de mudar a data da consulta em caso de necessidade, 43,6\% diz que lhe é dado conhecimento, ao contrário de 56,5\% que afirma 
desconhecimento, contudo, 47,2\% diz que as consultas decorrem à hora marcada.

A perceção dos utentes sobre a funcionalidade familiar são bastante positivas, uma vez que $83,8 \%$ manifesta ter uma família altamente funcional, $14,2 \%$ moderadamente funcional e apenas $2 \%$ do total dos inquiridos perceciona famílias com disfunções acentuadas.

Relativamente à satisfação global, os valores possíveis da escala vão de 55 (min) a 275 (máx) e quanto maior for o valor obtido, maior será a satisfação dos utentes. Assim, verificamos que os dados oscilam entre um valor mínimo de 63 e um máximo de 275, correspondendo-lhe uma média de 218,92 um Dp de 46,29 e um CV de 21,14\%, o que nos indica também a existência de uma moderada dispersão em torno da média. As mulheres estão mais satisfeitas $(M=220,65)$ do que os homens $(M=220,65)$, porém as diferenças estatísticas não são significativas $(\mathrm{p}=0,809)$ (Cf. Tabela 1$)$.

\section{Tabela 1}

Estatísticas descritivas da satisfação global dos Utentes por género

\begin{tabular}{c|c|c|c|c|c|c|c}
\hline $\begin{array}{c}\text { Satisfação } \\
\text { global }\end{array}$ & Min & Máx & Media & Dp & Sk & $\mathbf{K}$ & $\begin{array}{c}\text { CV } \\
(\%)\end{array}$ \\
\hline Masculino & 83 & 275 & 214,80 & 51,54 & $-2,36$ & 0,03 & 23,99 \\
\hline Feminino & 63 & 275 & 220,65 & 44,06 & $-5,69$ & 4,48 & 19,96 \\
\hline Amostra total & 63 & 275 & $\mathbf{2 1 8 , 9 2}$ & 46,29 & $-5,83$ & 3,02 & 21,14 \\
\hline
\end{tabular}

Numa análise global por dimensões (variando cada uma delas numa escala de 0-100), podemos afirmar que o grau de satisfação dos utentes é elevado, contudo e considerando os valores máximos possíveis em cada dimensão, constatamos que o grau de satisfação dos utentes é maior no item relação/comunicação (85,6\%), seguindo-se a informação e apoio $(84,1 \%)$, na terceira posição os cuidados médicos $(82,6 \%)$, na quarta posição a continuidade /cooperação (82,5\%), e por último a dimensão com menor satisfação diz respeito à organização dos serviços $(76,4 \%)$.

Os dados relativos à satisfação dos utentes com os cuidados (dimensões) da equipa de Enfermagem é também bastante positivo, sendo a dimensão interpessoal a mais apreciada (89,9\%), seguindo-se a relação de ajuda (88,8\%), e por fim a dimensão instrumental com 79,9\%.

A análise inferencial do estudo sobre as correlações existentes entre as variáveis mostrou que os utentes mais satisfeitos eram os que auferiam de maior rendimento mensal $(\mathrm{p}=0,002)$, os que reconheciam maiores benefícios aos programas de reabilitação $(\mathrm{p}=0.004)$ e percecionavam famílias altamente funcionais $(\mathrm{p}=0.035)$. 
Inversamente o género, a idade, as habilitações académicas e a situação profissional não se correlacionam de forma significativa $(p>0,05)$ com a satisfação dos utentes com o Centro de Saúde.

\section{DISCUSSÃO DOS RESULTADOS}

A satisfação dos utentes com os serviços representa na atualidade um indicador de qualidade fundamental constituindo-se o eixo motivador que nos conduziu ao presente estudo.

Verificámos que a satisfação geral dos utentes deste Centro de Saúde é de um modo geral bastante positiva com os serviços prestados. Contudo, verificámos que o grau de satisfação dos utentes é mais elevado nas dimensões relação/comunicação (85,6\%), informação e apoio $(84,1 \%)$, nos cuidados médicos (82,6\%), e na continuidade /cooperação (82,5\%). Inversamente, a dimensão que reflete menor satisfação diz respeito à organização dos serviços (76,4\%). Ferreira (2014) apresentam resultados idênticos a este estudo revelando que os utentes se apresentam bastante satisfeitos com a globalidade dos itens.

No caso específico da prestação de serviço pela Equipa de Enfermagem constatámos que de um modo geral o grau de satisfação dos utentes é também bastante positivo: assim, a satisfação é maior na dimensão interpessoal (89,9\%), seguindo-se a relação de ajuda (88,8\%), na terceira e última posição está a dimensão instrumental $(79,9 \%)$. Afirmam que uma das "surpresas agradáveis" na prestação de serviços do Centro de Saúde corresponde aos cuidados de enfermagem, considerando os Enfermeiros "pessoas extraordinárias". A implementação do enfermeiro de família (efetivado neste centro) promove o relacionamento e faz com que este profissional esteja cada vez mais próximo dos utentes. Já em relação às condições do Centro de Saúde, a satisfação dos utentes continua a ser positiva, no entanto é maior ao nível das respostas dadas $(84,6 \%)$, da atenção disponibilizada $(83,6 \%)$, da qualidade das instalações $(83,1 \%)$ e pontualidade $(81,4 \%)$. A pior classificação (traduzindo-se em menor satisfação) recai na dimensão "serviços prestados".O estudo levado a cabo por Oliveira (2012) demonstra aspectos um pouco diferentes, uma vez que o maior grau de insatisfação se relaciona com "o tempo de espera para a consulta” assim como á "acessibilidade ao centro de saúde

Os "aspetos a melhorar no centro de saúde" sugeridos pelos nossos participantes foram variados, contudo destacam-se por ordem de importância, o não pagar taxas; melhorias no atendimento telefónico; 
maior compreensão por parte da classe médica; mais conforto (ao nível das cadeiras, da temperatura, e do ruído) e melhor equipamento áudio. São dados que vem corroborar os resultados de Godinho (2011) ao referir as questões ambientais e de instalações como mais suscetiveis de desagrado dos utentes.

As correlações estabelecidas entre variáveis, neste estudo, confirmam (com significância estatística) que a satisfação dos utentes com o Centro de Saúde é mais elevada: nos indivíduos com maiores rendimentos mensais, ou seja, naqueles que auferem valores acima de 500 euros, naqueles que percecionam maior funcionalidade familiar e ainda nos utentes que percepcionam maiores benefícios no desenvolvimento dos programas de reabilitação.

De modo inverso, o género, a idade, as habilitações académicas, a situação profissional e o realizar programas de reabilitação, mostraram não exercer influencia na satisfação destes Utentes. Estes resultados são similares aos de Cerqueira (2012) quando afirma que a satisfação dos utentes é influenciada por variados factores contudo o genero e a idade não tem relação direta com a satisfação.

\section{CONCLUSÕES}

O conhecimento constante e actualizado, apenas é possível através da realização de estudos de investigação nas diversas áreas científicas. A saúde não é excepção, porém tem merecido maior destaque a Satisfação dos Utentes com os Serviços uma vez que representam um indicador de qualidade.

Foi este o eixo motivador que nos conduziu á presente investigação e portanto em jeito de síntese final, e debruçando-nos sobre o percurso investigativo realizado até aqui, com foco nos principais objectivos concluímos:

Que a satisfação geral dos utentes do Centro de Saúde é de um modo geral bastante positive, sendo mais elevada nas dimensões relação/comunicação, informação e apoio, nos cuidados médicos, e na continuidade /cooperação. Inversamente a dimensão que reflecte menor satisfação diz respeito à organização dos serviços.

Deste modo torna-se imperioso investigar sobre o nível de satisfação dos utentes de modo a poder-se promover alterações e/ou reajustes necessários aos cuidados prestados, para satisfazer mais e melhor aqueles que recorrem aos serviços dos centros de saúde, tornando os mesmos, um local de excelência nos cuidados prestados. Isto obviamente, apenas será conseguido se todos os profissionais da instituição em geral estiverem 
sensibilizados para a necessidade de garantir a qualidade dos cuidados e avaliar/monitorizar continuamente os graus de satisfação dos utentes sobre os cuidados recebidos. É igualmente fundamental ter a perceção dos pontos fortes e fracos que possam influenciar essa satisfação, no sentido de desenvolver estratégias de mudança que conduzam aos ganhos em saúde.

\section{FONTES E BIBLIOGRAFIA}

Academia das Ciências de Lisboa, \& Fundação Calouste Gulbenkian. (2001). Dicionário da língua portuguesa contemporânea da Academia das Ciências de Lisboa. Lisboa: Verbo.

Arakawa, Y., Shibata, N., Shibayama, K. Kurokawa, H., Yagi, T., Fujiwara, H., \& Goto, M. (2012). Convenient test for screening metallo- $\beta$-lactamase-producing gram-negative bacteria by using thiol compounds. Journal of Clinical Microbiology, 38, 40-43. Acedido em https://www.ncbi.nlm.nih.gov/pmc/articles/PMC86013/

Cerqueira, A. R. S. M. (2012). Cuidados de enfermagem numa USF de Ponte de Lima: Satisfação dos utentes (Projecto final de licenciatura, Universidade Fernando Pessoa). Acedido em http://hdl.handle.net/10284/3502

Costa, G. B. (2011). Avaliação da satisfação dos utentes do hospital de dia de especialidades médicas do Hospital de Egas de Moniz (Dissertação de mestrado, Universidade Nova de Lisboa). Acedido em http://hdl.handle.net/10362/9257

Duarte, A. R. C. (2012). Satisfação com a informação nos utentes com cardiopatia isquémica (Dissertação de mestrado, Instituto Politécnico de Viseu). Acedido em http://hdl.handle.net/10400.19/1691

Ferreira, C. A. S. (2014). Satisfação dos utentes da Unidade Local de Saúde do Nordeste face aos cuidados de enfermagem (Dissertação de mestrado, Instituto Politécnico de Bragança). Acedido em http://hdl.handle.net/10198/9785

Ferreira, P. L., Raposo, V., \& Godinho, P. (2005). A voz dos utilizadores dos centros de saúde. Lisboa: Instituto da Qualidade em Saúde.

Godinho, A. C. S. (2011). Qualidade, satisfação e lealdade nos serviços de saúde (Dissertação de mestrado, Universidade de Aveiro). Acedido em http://hdl.handle.net/10773/8525

Lopes, S. M. G. M. (2013). Satisfação dos utentes com os cuidados de enfermagem na unidade de cuidados de saúde personalizados de 
Eiras (Dissertação de mestrado, Escola Superior de Enfermagem de Coimbra). Acedido em http://repositorio.esenfc.pt/?url=KaQehi

Marques, T. P. S. (2010). Da qualidade à satisfação do utente: Serviço de colheita de sangue e seu contributo para a satisfação do utente (Dissertação de mestrado; Universidade de Trás os Montes e Alto Douro). Acedido em http://hdl.handle.net/10348/1416

Oliveira, António José Eugénio. (2012). Satisfação dos utentes numa unidade de saúde do interior (Dissertação de mestrado, ISCTE Instituto Universitário de Lisboa). Acedido em http://hdl.handle.net/10071/5027

Ordem dos Enfermeiros. (2011). Regulamento dos padrões de qualidade dos cuidados especializados em enfermagem de reabilitação: Proposta do Conselho Diretivo após aprovação por unanimidade com aclamação em assembleia de colégio de especialidade de enfermeiro de reabilitação realizado no dia 30 de Setembro de 2011. Acedido em http://www.ordemenfermeiros.pt/colegios/documents/pqceereabilitaca o.pdf

Vilares, M., \& Coelho, P. (2005). A satisfação e lealdade do cliente: Metodologias de gestão, avaliação e análise. Lisboa: Escolar Editora. 